\title{
Novel egg white-based 3-D cell culture system
}

\author{
Benny Abraham Kaipparettu1, Isere Kuiatse1, Bonita Tak-Yee Chan1, Meju Benny Kaipparettu², \\ Adrian V. Lee', and Steffi Oesterreich ${ }^{1}$
}

BioTechniques 45:165-171 (August 2008)

doi $10.2144 / 000112883$

\begin{abstract}
Although three dimensional (3-D) cell culture systems have numerous advantages over traditional monolayer culture, the currently available 3-D cell culture media are cost-prohibitive for regular use by the majority of research laboratories. Here we show a simple system based on avian egg white that supports growth of cells in 3-D, at a significantly decreased cost. Specifically, we show that growth of immortalized human breast epithelial cells (MCF10A) in egg white-based medium results in formation of acini with hollow lumens, apoptotic clearance of the cells in the lumen, and apicobasal polarization comparable to what has been described using established 3-D culture media such as reconstituted basement membrane preparations (BM). There was no significant difference in MCF10A proliferation and acinar size between egg white and BM. We also cultured different established cell lines, oncogene-transformed MCF10A, and mouse mammary epithelial cells in egg white and BM, and observed similar morphology. In summary, our data convincingly argue that egg white can be used as a suitable alternative model for 3-D cell culture studies. We strongly believe that this simple and inexpensive method should allow researchers to perform 3-D cell culture experiments on a regular basis, and result in a dramatic increase of use of the 3-D cell culture in research. Thus, this finding lays the foundation for significantly increased, cost-effective use of 3-D cultures in cell biology.
\end{abstract}

\section{INTRODUCTION}

Over the last few years, much attention has been paid to the development and use of three dimensional (3-D) cell culture systems in order to replace the classical two-dimensional (2-D) monolayer system. Cells grown on flat 2-D tissue culture substrates can differ considerably in their morphology, differentiation, and cell-cell and cell-matrix interactions from those growing in more physiological 3-D environments (1). There are a growing number of studies that report differences in phenotype, cellular signaling, cell migration, and drug responses when the same cells are grown under 2-D or 3-D culture conditions (2). 3-D cultures are currently used in a broad range of cell biology studies, including tumor biology, cell adhesion, cell migration, and epithelial morphogenesis (3). Commonly used 3-D culture media contain a reconstituted basement membrane preparation derived from Engelbreth-Holm-Swarm (EHS) sarcoma, a mouse tumor rich in extracellular matrix (ECM) proteins. Unfortunately, the cost of all currently available 3-D media is extremely high, which prohibits their frequent use in the majority of academic research settings.

In our search for a reliable and economically viable replacement for the expensive commercial 3-D media, we hypothesized that avian egg white could be a potential alternative based on the following ratio- nales: (i) It is sufficient to support growth and development of normal cells into a baby chick without any additional external nutrient support; (ii) the viscosity of egg white can be changed with temperature; (iii) egg white is transparent, which allows easy monitoring of growing cells; and (iv) it is inexpensive and easily available.

To demonstrate that egg white can indeed function as ECM and support growth of cells in 3-D, we used the nontransformed immortalized human breast epithelial cell line MCF10A as an example, since its growth in 3-D has been very well characterized. In 3-D, MCF10A cells recapitulate numerous features of normal breast epithelium in vivo, including the formation of spheroids resembling acini with a hollow lumen, and apical-basal polarization (4). This is in stark contrast to fully transformed epithelial cells, which do not develop tightly organized acinar structures but instead form large structures that lack organized adherent junctions and a central lumen and that fail to undergo growth arrest (5).

\section{MATERIALS AND METHODS}

\section{Preparation of Egg White-based Chamber Slide}

We purchased chicken eggs from grocery stores or directly from the farm. After cleaning the outer shell of the egg
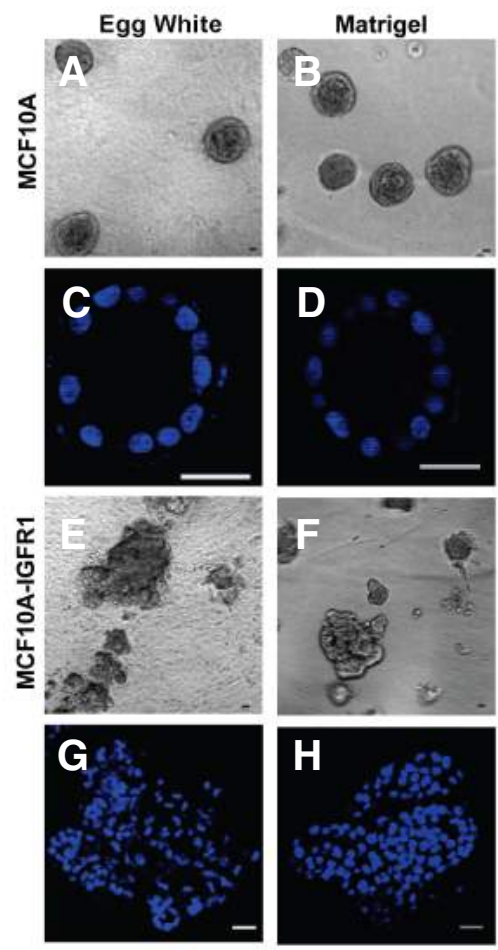

Figure 1. MCF10A cell growth in egg white and Matrigel. Phase contrast micrographs (10X) of the gross morphology of MCF10A cells grown for 8 days in egg white-based (A) and Matrigel-based (B) 3 -D culture. Respective $40 \times$ confocal images with TOPRO3 nuclear staining of MCF10A acini after 12 days culture are shown in (C) and (D). Phase contrast micrographs (10x) of MCF10A-IGFR1 cells grown for 8 days in egg white (E) and Matrigel $(\mathrm{F})$. The corresponding confocal images in $40 \times$ after 12 days are $\mathrm{G}$ and $\mathrm{H}$. Cells were grown as described in the text. The scale bar is $25 \mu \mathrm{m}$. 


\section{Research Reports}

using $70 \%$ alcohol, we made a small hole in the shell using sterilized metal forceps or a sterile pipet under a laminar flow hood. We then poured the egg white into a $50 \mathrm{ml}$ falcon tube to ensure complete separation from egg yolk. To prevent the egg yolk from mixing with egg white, it is important to avoid removing the egg white completely from the shell. From one chicken egg, approximately 25 to $30 \mathrm{ml}$ of egg white can be obtained. Egg white can be aliquoted into $1.5 \mathrm{ml}$ sterile microcentrifuge tubes and stored at $-80^{\circ} \mathrm{C}$ until use.

For cell culture, we thawed the egg white to room temperature and pipeted $100 \mu \mathrm{l}$ using a $1000 \mu \mathrm{l}$ pipet tip into each well of an 8-well chamber slide. Because the egg white is very viscous and it may be difficult to pipet with a normal sharp pipet tip, cutting the tip may be helpful. The egg white should be added directly to the middle of the wells, using caution that the egg white does not touch the top of the side wall of the wells. Spilling of egg white on the top of the side walls may cause capillary rise of the medium from the well to the cover of the chamber through the side walls, which may result in drying of the wells and contamination.

We then heated the 8-well chamber containing the egg white at $60^{\circ} \mathrm{C}$ on a heating block of the type used for $1.5 \mathrm{ml}$ microcentrifuge tubes for 30-60 min. We found that there should be a minimum of direct contact between the wells and the metal part of the heating block to avoid rapid heating of some areas of the chamber; thus, the slide should be positioned in such a way that the chamber wells mostly align with the wells of the heating block and are not directly on the metal part. Increased temperature may lead to denaturing of the protein, causing the egg white to become white in color. If this occurs, the affected chambers should not be used in subsequent experiments. Evaporated water from the egg white usually condenses on the chamber cover, so at regular intervals we would shake off the condensed water from the chamber cover under a sterile hood. After approximately 30-60 $\mathrm{min}$, the egg white becomes a semi-solid that adheres to the bottom of the well. The time varies depending on the heating block and the thickness of the egg white. When most of the egg white had adhered to the chamber slide, we took the slide back to the sterile hood and brought it back to room temperature. It is important to remove the chamber from the heating block before the entire egg white becomes semi-solid because this may cause drying of the egg white in some areas and formation of a round dry area on which cells are not able to grow in the middle of the chamber. We washed out any unstuck egg white from the chamber by adding 500 $\mu \mathrm{l}$ of growth medium to the chamber and removing it slowly using a Pasteur pipet. This step is important because the cells could grow on the unstuck egg white and will be lost later while changing the medium. We would either use the chamber right away for cell culture, or store it at $4^{\circ} \mathrm{C}$ overnight after adding $0.5 \mathrm{ml}$ of sterile $\mathrm{PBS}$ or growth medium to each well.

\section{Cell Culture}

We trypsinized MCF10A cells, diluted the cell suspension to $4 \times 10^{3}$ cells $/ \mathrm{mL}$, scan Scan Better with the FLA-7000.

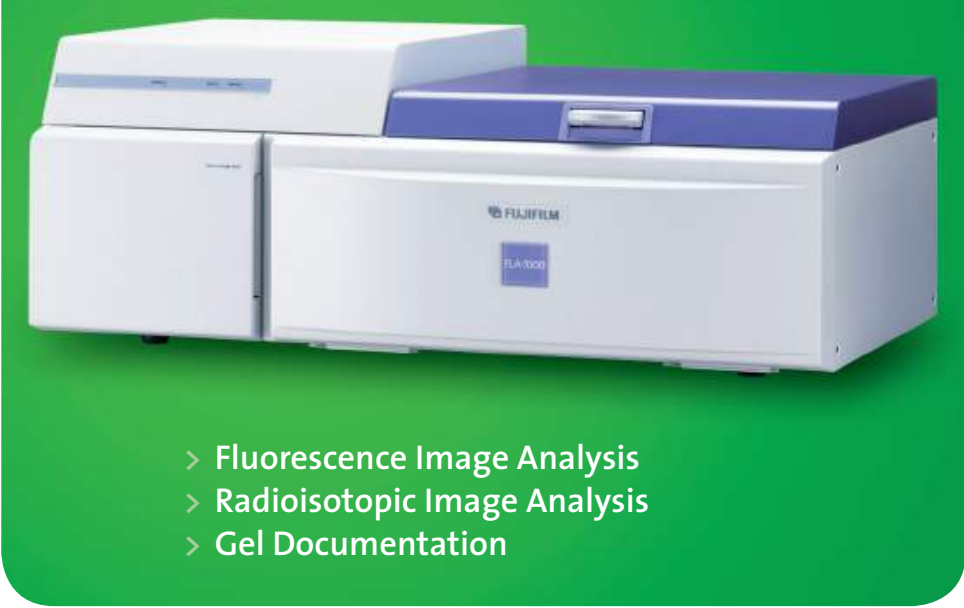

Fujifilm's FLA-7000 is the better choice

in imaging systems for fluorescence and radioisotopic image analysis and gel documentation. With four easy-to-access filters and four different excitation laser beams, the FLA-7000 offers more flexibility than any other system.

All this, plus the FLA-7000 can complete scans $85 \%$ faster than the competition, takes half the space, and costs half as much.

\section{Do it Better with Fujifilm's Imaging Systems}
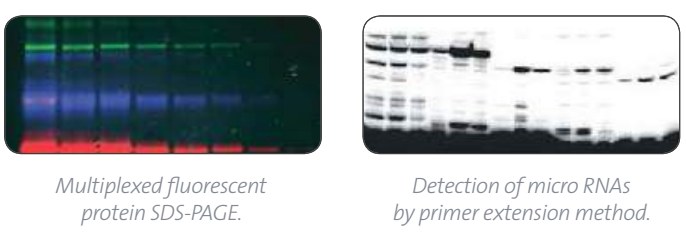

\section{FUJifILM Life Science}



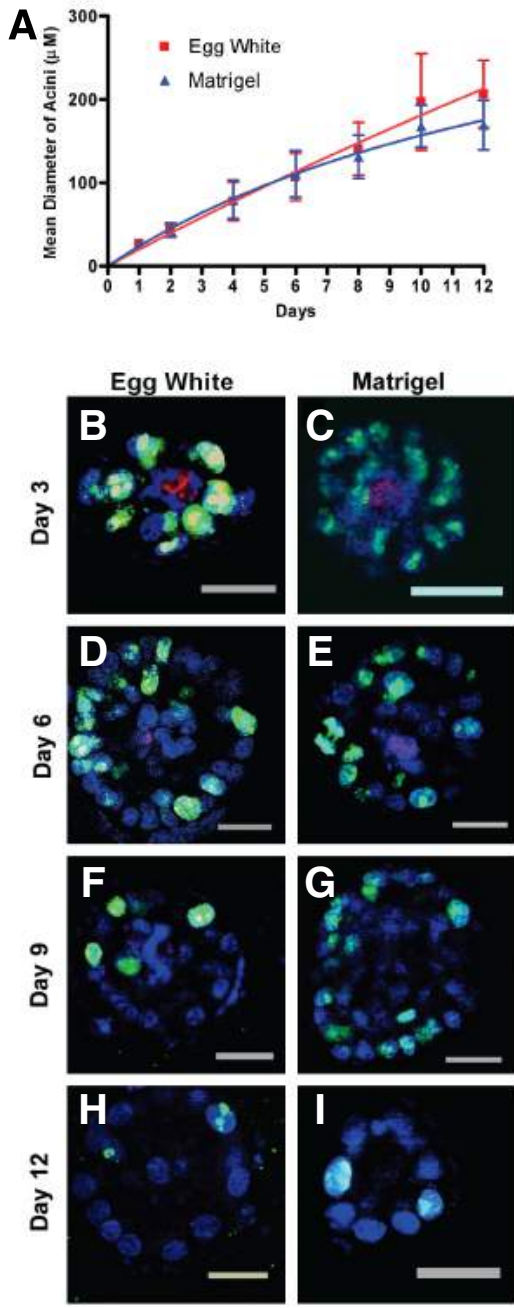

Figure 2. Cell growth and proliferation of MCF10A cells. Growth of MCF10A acini in egg white and Matrigel at Day 1, 2, 4, 6, 8, 10, and 12. Mean acini diameter \pm SD on each day is plotted (A). MCF10A cells were cultured in egg white for $3(\mathrm{~B}), 6(\mathrm{D}), 9(\mathrm{~F})$, and $12(\mathrm{H})$ days and examined for caspase-3 (red) and Ki67 (green) by immunofluorescence. Corresponding pictures are shown for cells grown in Matrigel (C, E, G, and I, respectively). Nuclei were counterstained with TOPRO3 (blue) and photographed on a Nikon Eclipse E1000 confocal microscopy system $(40 \times)$. The scale bar is $25 \mu \mathrm{m}$.

and added $0.5 \mathrm{ml}$ of the cell suspension directly into each well. Cells are cultured in $5 \% \mathrm{CO}_{2}$ at $37^{\circ} \mathrm{C}$ and should be regularly monitored for cell growth, changing the growth medium every 3 days till Day 6 and every other day after that. The growth medium used was according to the previously published protocol for MCF10A cells by Debnath et al. (4) except that we used the growth medium for all the steps. For comparative studies, we used a well-established reconstituted basement membrane matrix preparation (Matrigel obtained from
BD Bioscience Pharmingen, San Jose, CA, USA). Chamber slides with Matrigel were prepared as described in J. Debnath et al. (4) except that we used $80 \mu \mathrm{l}$ of Matrigel. For both egg white and Matrigel-based overlay methods, we used growth medium, with the addition of 2\% Matrigel. Of note, we also performed most of the egg whitebased studies omitting the Matrigel in the overlay media, and did not observe any significant differences in the phenotypes we analyzed. In addition, we cultured insulin-like growth factor receptor 1 (IGFR1)-transformed MCF10A cells (MCF10A-IGFR1) (6), different established human cell lines (MCF7, HEK293, HeLa, LNCaP, and Saos-2), and mouse mammary tumor virus-polyoma middle $\mathrm{T}$ antigen (MMTV-PyMT)-transformed mouse mammary epithelial cells (kindly provided by Jianming $\mathrm{Xu}$ ) using the same culture protocol described above but in their appropriate growth media.

\section{Acinar Growth and Apoptosis Analysis}

For growth analysis we compared the size gross morphology of MCF10A acini grown in egg white to that grown in Matrigel. MCF10A cells were grown in egg white or Matrigel in 8-well culture chambers as described in the Cell Culture section. Phase contrast pictures at $10 \times$ magnification were taken after 1, 2, 4, 6, 8 , and 12 days and the diameter of each acinus was measured from two different angles using CellProfiler image analysis software (www.cellprofiler.org) (7). The measurements in pixels were converted into micrometers. An average of 50 acini were measured each day (22 to 83 acini per day). Live and apoptotic cells were analyzed using calcein AM-ethidium homodimer III (EthD-III) (Biotium, Inc., Hayward, CA, USA) staining as previously published (8). In brief, after removing assay medium, acini were washed once with PBS and incubated for $15-30 \mathrm{~min}$ at $37^{\circ} \mathrm{C}$ with a mixture of calcein AM-EthD-III in PBS, and indirect immunofluorescence pictures were taken. An Olympus IX70 fluorescence microscope (Olympus, Center Valley, PA, USA) was used for these experiments.

\section{Confocal Immunofluorescence Analysis}

The immunofluorescence analysis was carried out according to the protocol published by Debnath et al. (4) with the following modifications. We permeabi-
ANEW BENGHIMARK IN PIPETIES The BRAND Transferpetite $\mathcal{S}$

The new BRAND Transferpette ${ }^{\circledR} \mathcal{S}$ pipettes offer an array of performance and convenience features never before combined in a single pipette.

- Simple, thumb-tip volume adjustment

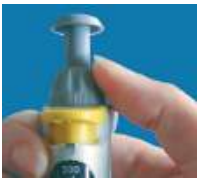

- Short pipetting stroke reduces fatique

- Covered I.D. label

- Fast, simple disassembly for in-house cleaning, service

- Built-in calibration without tools

- Completely autoclavable

- Models for $0.1 \mu \mathrm{L}$ to $10 \mathrm{~mL}$

Buy 3, get 1 FREE! Promotion details at www.brandtech.com/3-1free

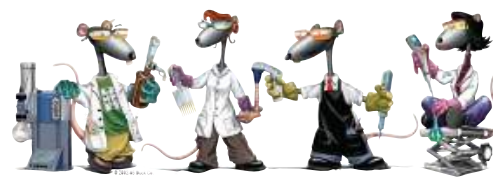

Lab Rats Trust BrandTech!

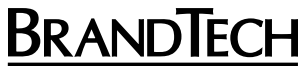
SCIENTIFIC, INC.

Toll Free (888) 522-2726 www.brandtech.com 


\section{Research Reports}
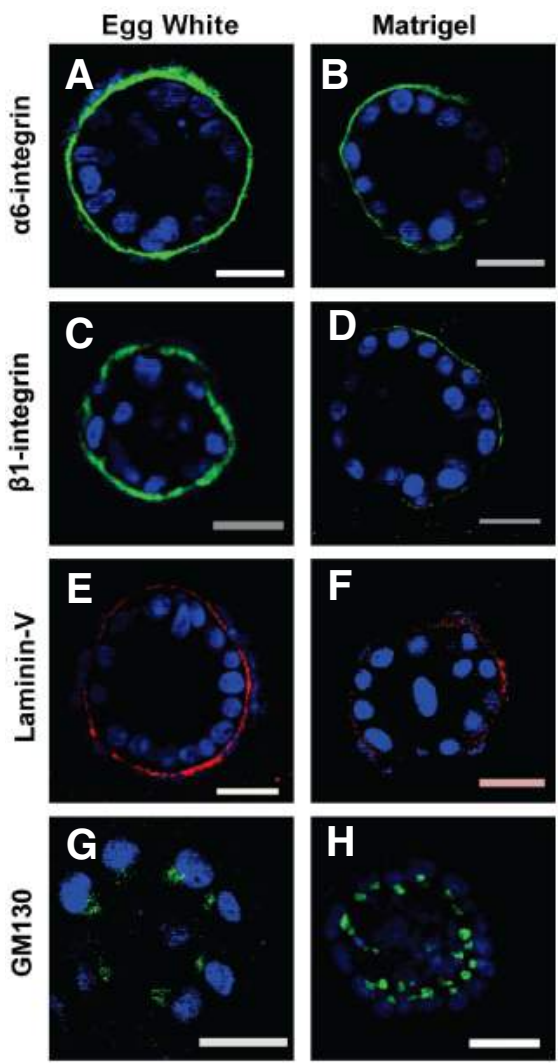

Figure 3. Apicobasal polarization of MCF10A cells. MCF10A cells were cultured in egg white for 12 days and stained with $\alpha 6$-integrin in green (A), $\beta 1$-integrin in green $(C)$, laminin- $V$ in red (E), and GM130 in green (G) immunofluorescence. The respective cultures in Matrigel are shown in B, D, F, and H. Nuclei were counterstained with TOPRO3 (blue) and photographed on a Nikon Eclipse E1000 confocal microscopy system $(40 \times)$. The scale bar is $25 \mu \mathrm{m}$.

lized the cells at room temperature for 40 min and performed one-step blocking with IF medium containing $10 \%$ horse serum. The primary antibodies were diluted to $1: 100$ in a freshly prepared IF buffer with $10 \%$ horse serum. Primary antibodies used were cleaved caspase-3 (Cell Signaling Technology, Danvers, MA, USA), $\alpha 6$ and $\beta 1$ integrin (BD Biosciences), laminin-V (Chemicon, Temecula, CA, USA), GM130 (BD Biosciences), and Ki67 (Dako, Carpinteria, CA, USA). The secondary antibodies used were Alexa fluor antibodies at 1:200 dilution (Molecular Probes, Eugene, OR, USA). Nuclear staining was performed by incubation for 15 min with PBS containing $5 \mu \mathrm{M}$ TOPRO-3 (Molecular Probes) before cells were mounted with the antifade agent VECTASHIELD (Vector Laboratories, Burlingame, CA, USA). Confocal analyses were performed with a Nikon Eclipse
E1000 (Nikon USA, Melville, NY, USA) confocal microscopy system.

\section{RESULTS}

The dichotomy between 2-D and 3-D cell culture is highlighted by the investigation of epithelial morphogenesis (3). The mammary gland provides an ideal model for understanding relationships between normal tissue function and architecture (9). 3-D culture of mammary epithelial cell lines such as MCF10A has been extensively used to investigate how the biochemical and physical properties of the ECM drive morphogenesis and homeostasis of mammary epithelial acini (3). When cultured on reconstituted basement membrane, MCF10A cells form spherical structures (acini) in which a layer of polarized epithelial cells surrounds a hollow lumen, resembling glandular epithelium in vivo $(4,10)$.

To test the growth of acini in egg white, we cultured MCF10A cells in egg white and Matrigel side by side. As shown in Figure 1, gross morphology of MCF10A cells grown in egg white and Matrigel was indistinguishable-both model systems resulted in growth of spheroidlike organized structures (Figure 1, A and B). Importantly, confocal microscopy revealed that cells grown in egg white were able to form acini with a hollow lumen in the middle (Figure 1C), as observed with Matrigel (Figure 1D). It is known that the intact, well-ordered architecture of epithelial cells is disrupted during the pathogenesis of epithelial tumors (10). Thus we cultured MCF10A-IGFR1 cells with a constitutively active IGFR 1 oncogene (6) in both egg white and Matrigel. As expected, disregulated architecture was observed with MCF10A-IGFR1 in both egg white and Matrigel (Figure 1, E and F). The confocal pictures reveal the multiacinar phenotype and luminal filling of MCF10A-IGFR1 in both systems (Figure $1, \mathrm{G}$ and $\mathrm{H})$. We also tried egg white from the peacock egg using the same protocol to evaluate whether the 3-D growth of MCF10A cells is restricted only to chicken egg white. As shown in Supplementary Figure S1 (see Supplementary Material available online at www.BioTechniques. com), the peacock egg white also supported the growth of MCF10A acini. In addition we used culture chamber of different sizes, and also thawed egg white after storage at $-80^{\circ} \mathrm{C}$ for various lengths of time (up to 6 months), and obtained similar results (data not shown).

We next compared the growth of MCF10A acini in egg white to that in Matrigel. Diameters of an average of 50 acini were measured each day from both egg white and Matrigel cultures at 1, 2, $4,6,8,10$, and 12 days post-culture, and the mean diameters were calculated. As shown in Figure 2A, the acinar size of MCF10A between egg white and Matrigel was comparable. It is known that when MCF10A cells are cultured in Matrigel, the proliferation marker Ki67 is prevalent during the early phases of acinus formation and decreases at later stages as acini increase in size and slow in their growth (11). Thus we compared the cell proliferation $\mathrm{M}$ between egg white and Matrigel cell culture systems. As shown in Figure 2 , B-I, the number of proliferating cells in MCF10A acini considerably decreased from Day 3 to Day 12 in both systems. Specifically, we counted 177 (Day 3), 417 (Day 6), 149 (Day 9), and 199 (Day 12) nuclei of MCF10A acini grown in Matrigel and observed $87 \%, 61 \%, 30 \%$, and $9.5 \%$ of Ki67-positive cells, respectively. Similarly we observed $80 \%, 53 \%, 30 \%$, and $5.6 \%$ of Ki67-positive cells when we counted 156 (Day 3), 141 (Day 6), 367 (Day 9), and 173 (Day 12) nuclei, respectively, in egg white showing a comparable proliferation pattern in both systems (see Supplementary Figure S2).

Studies have shown that apoptosis has a primary (but not an exclusive) role in acinar lumen formation by selectively clearing the cells that are located internally (3). To analyze apoptotic clearance of acini grown in egg white, we costained the apoptotic marker cleaved caspase-3 (Figure 2, B-I) and confirmed the apoptotic cells in the lumen of MCF10A cells cultured in both egg white and Matrigel. We observed more apoptotic cells in the lumen during the early days, which decreased gradually with acinar growth in both systems. We also performed calcein AM-EthD-III staining to further confirm the apoptotic cells in the lumen. Supplementary Figure S3 shows that, as reported in Matrigel (8), the dying ethidium bromide-positive cells were found only in the interior of egg whitecultured acini. However, outer cells, which are in direct contact with matrix, were alive and stained positive for calcein AM. Thus apoptosis is involved in clearing of the lumen of MCF10A in the egg white-based cell culture system.

Next we analyzed the apicobasal polarization of the acini in egg white 


\section{Research Reports}
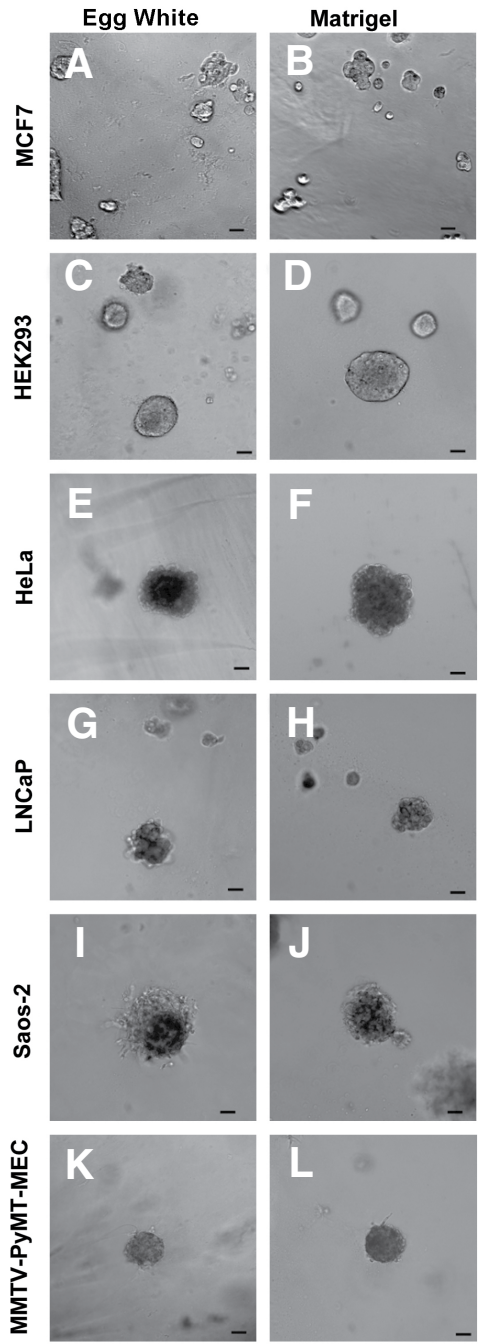

Figure 4. Egg white supports 3-D growth of various cells. Phase contrast micrographs were taken at 10× after MCF7 (A), HEK293 (C), $\mathrm{HeLa}(\mathrm{E}), \mathrm{LNCaP}(\mathrm{G})$, and Saos-2 (I) cells were cultured in egg white. MCF7 was photographed after 8 days and other cell lines after 12 days in culture. Corresponding images also taken after culture in Matrigel (B, D, F, H, and $\mathrm{J}$, respectively). MMTV-PyMT-transformed mouse mammary epithelial cells (MEC) were also cultured in egg white (K) and Matrigel (L) and photographed after 12 days. Olympus IX70 fluorescence microscope $(10 \times)$ used for imaging. The scale bar is $50 \mu \mathrm{m}$

It is known that, in addition to forming acini with hollow lumens, epithelial cells like MCF10A grown in 3-D cultures in reconstituted basement preparations like Matrigel also develop apicobasal polarization and establish their own basement membrane (10). Previous studies showed that, in polarized epithelia, $\alpha 6$ and $\beta 1$ integrin as well as laminin- $\mathrm{V}$ are located on the basal surface of the acini, whereas the cis-Golgi protein GM130 assumes an apical location (10). We therefore monitored the apicobasal polarization of cultured MCF10A acini by immunofluorescence of these four proteins. As shown in Figure 3, as in Matrigel, MCF10A cells cultured in egg white formed wellorganized acinar structures with apicobasal polarity.

To make sure that 3-D cell growth in egg white is not restricted to MCF10A cells, we then compared the growth of five other established human cell lines from different tissues: breast cancer cell line MCF7 (Figure 4, A and B), human embryonic kidney cell line HEK293 (Figure 4, C and D), human cervical cancer cell line HeLa (Figure 4, E and F), human prostate adenocarcinoma cell line LNCaP (Figure 4, G and H), and human osteosarcoma cell line Saos-2 (Figure 4, I and J), in egg white and Matrigel. As shown in Figure 4, A-J, all the cell lines tested showed comparable morphology in both 3-D cell culture systems. We also cultured MMTV-PyMT-transformed mouse mammary epithelial cells in egg white and Matrigel. As shown in Figure 4, egg white (Figure 4K) did support the growth of the mouse mammary tumor cells as well as Matrigel (Figure 4L). Thus the use of egg white as a 3-D cell culture medium is not tissue or species restricted.

\section{DISCUSSION}

The present study shows that avian egg white can support the 3-D growth of human and mouse cells, which is comparable with established $3-\mathrm{D}$ cell culture media. The proteins in egg white have very different molecular masses (1.27-800 $\mathrm{kDa})$. Their concentration also varies widely, with ovalbumin representing more than $50 \%$ of total proteins, thus possibly making detection of minor proteins very difficult. These reasons probably explain why very little research has been devoted to the identification and characterization of the minor egg white proteins, even though they probably play essential roles with respect to the original biological action of egg white, including embryo protection and development (12).

Recent progress in egg white protein analysis has identified many novel proteins (12). There are also some as of yet unidentified proteins in the egg white. Some of these known or unknown proteins are probably essential for the function of egg white as a supporting matrix for the embryo development and also in 3-D cell growth. Ovalbumin itself has been described as an inert material that poorly supports cell attachments. However, ovalbumin is commonly used as a supporting factor in cell adhesion. For example, covalent linkage of biologically active peptides to proteins like ovalbumin is an efficient way to immobilize peptides as cell adhesion substrata (13). Some of the minor proteins in egg white are important for their possible role as an extracellular matrix. For example, cystatin was one of the first cysteine peptidase inhibitors (CPIs) identified and may have an important role in cancer metastasis (14). In addition, cystatin $\mathrm{C}$ acts as a TGF- $\beta$ receptor antagonist and is capable of inhibiting TGF- $\beta$ signaling. Since TGF- $\beta$ is a known regulator of integrins, cystatin may also have a role in the regulation of integrins, the major receptors for the extracellular matrix (15).

Another important egg white protein is avidin. Avidin contains the Arg-TyrAsp-Ser (RYDS) sequence, which mimics the Arg-Gly-Asp-Ser (RGDS) sequence in the cell-wall recognition site of extracellular matrix proteins. Since the RGD sequence has been shown to play a key role in integrin-mediated cell adhesion (16), it is possible that avidin may use the RYDS site to regulate cell adhesion. There might be other factors in egg white that also may play critical roles in supporting the cell's 3-D growth. However, further analysis will be necessary to identify such critical factors.

The topography of the ECM is characterized by features over different length scales ranging from the nano- to the mesoscale, and the ECM regulates cellular behavior in a way that it is still far from completely understood (17). Materials other than the conventional reconstituted basement membrane matrix preparation have also been reported to support 3-D cell culture. For example, Carbone et al. (17) reported cluster-assembled nanostructured titanium dioxide surfaces as a biocompatible surface for cell culturing. Jayawarna et al. (18) have used a set of peptides to create a 3-D peptide hydrogel that mimics the extracellular matrix in supporting cell growth. Similarly, alginate scaffolds have been developed for 3-D cell culture (19).

At present the commercially available 3-D cell culture media, including the reconstituted basement membrane preparations, are economically unviable for many research laboratories. Thus our effort was to identify an alternative material that can be reliably used as a 3-D cell culture 
Mutation Discovery, Genotyping

and Gene Scanning

medium, and the experiments described herein suggest that egg white is such a medium. Since egg white is inexpensive compared to the commercially available 3-D cell culture media, egg white-based 3 -D culture can drastically reduce the cost of 3-D research. Modified egg white preparations may yield an even more readily usable product for researchers.

\section{ACKNOWLEDGMENTS}

We thank Hyun-Jung Kim, Robert Dearth, Shiming Jiang, Beate Litzenburger, and Ora Britton for providing support and critical expertise for these studies. We also thank Gary Chamness for valuable comments on the manuscript.

\section{COMPETING INTERESTS STATEMENT}

B.K., M.K., and S.O. have filed a U.S. patent application pertaining to the eggbased 3-D culture system described herein. The other authors declare no competing interests.

\section{REFERENCES}

1. Yamada, K.M. and E. Cukierman. 2007. Modeling tissue morphogenesis and cancer in 3D. Cell 130:601-610.

2. Smalley, K.S., M. Lioni, and M. Herlyn. 2006. Life isn't flat: taking cancer biology to the next dimension. In Vitro Cell. Dev. Biol. Anim. 42:242247.

3. Pampaloni, F., E.G. Reynaud, and E.H. Stelzer. 2007. The third dimension bridges the gap between cell culture and live tissue. Nat. Rev. Mol. Cell Biol. 8:839-845.

4. Debnath, J., S.K. Muthuswamy, and J.S. Brugge. 2003. Morphogenesis and oncogenesis of MCF-10A mammary epithelial acini grown in three-dimensional basement membrane cultures. Methods 30:256-268.

5. Boudreau, N.J. 2003. Organized living: from cell surfaces to basement membranes. Sci. STKE 2003:pe34.

6. Kim, H.J., B.C. Litzenburger, X. Cui, D.A. Delgado, B.C. Grabiner, X. Lin, M.T. Lewis, M.M. Gottardis, et al. 2007. Constitutively active type I insulin-like growth factor receptor causes transformation and xenograft growth of immortalized mammary epithelial cells and is accompanied by an epithelial-to-mesenchymal transition mediated by NF-kappaB and snail. Mol. Cell. Biol. 27:3165-3175.

7. Carpenter, A.E., T.R. Jones, M.R. Lamprecht, C. Clarke, I.H. Kang, O. Friman, D.A. Guertin, J.H. Chang, et al. 2006. CellProfiler: image analysis software for identifying and quantifying cell phenotypes. Genome Biol. 7:R100.

8. Debnath, J., K.R. Mills, N.L. Collins, M.J. Reginato, S.K. Muthuswamy, and J.S. Brugge. 2002. The role of apoptosis in creating and main- taining luminal space within normal and oncogene-expressing mammary acini. Cell 111:29-40.

9. Sodunke, T.R., K.K. Turner, S.A. Caldwell, K.W. McBride, M.J. Reginato, and H.M. Noh. 2007. Micropatterns of Matrigel for three-dimensional epithelial cultures. Biomaterials 28:40064016.

10. Debnath, J. and J.S. Brugge. 2005. Modelling glandular epithelial cancers in three-dimensional cultures. Nat. Rev. Cancer 5:675-688.

11. Coppock, H.A., D.E. Gilham, A. Howell, and R.B. Clarke. 2007. Cyclin-dependent kinase inhibitors and basement membrane interact to regulate breast epithelial cell differentiation and acinar morphogenesis. Cell Prolif. 40:721-740.

12. Guerin-Dubiard, C., M. Pasco, D. Molle, C. Desert, T. Croguennec, and F. Nau. 2006. Proteomic analysis of hen egg white. J. Agric. Food Chem. 54:3901-3910.

13. LeBaron, R.G. and K.A. Athanasiou. 2000. Extracellular matrix cell adhesion peptides: functional applications in orthopedic materials. Tissue Eng. 6:85-103.

14. Saleh, Y., M. Siewinski, W. Kielan, P. Ziolkowski, M. Grybos, and J. Rybka. 2003. Regulation of cathepsin B and L expression in vitro in gastric cancer tissues by egg cystatin. J. Exp. Ther. Oncol. 3:319-324.

15. Sokol, J.P. and W.P. Schiemann. 2004. Cystatin $\mathrm{C}$ antagonizes transforming growth factor beta signaling in normal and cancer cells. Mol. Cancer Res. 2:183-195.

16. Alon, R., R. Hershkoviz, E.A. Bayer, M. Wilchek, and O.Lider. 1993. Streptavidin blocks immune reactions mediated by fibronectin-VLA-5 recognition through an Arg-Gly-Asp mimicking site. Eur. J. Immunol. 23:893-898.

17. Carbone, R., I. Marangi, A. Zanardi, L. Giorgetti, E. Chierici, G. Berlanda, A. Podesta, F. Fiorentini, et al. 2006. Biocompatibility of cluster-assembled nanostructured $\mathrm{TiO} 2$ with primary and cancer cells. Biomaterials 27:32213229.

18. Jayawarna, V., A. Smith, J.E. Gough, and R.V. Ulijn. 2007. Three-dimensional cell culture of chondrocytes on modified di-phenylalanine scaffolds. Biochem. Soc. Trans. 35:535-537.

19. Dvir-Ginzberg, M., I. Gamlieli-Bonshtein, R. Agbaria, and S. Cohen. 2003. Liver tissue engineering within alginate scaffolds: effects of cell-seeding density on hepatocyte viability, morphology, and function. Tissue Eng. 9:757-766.

Received 18 June 2007; accepted 23 March 2008.

Address correspondence to Steffi Oesterreich, Baylor College of Medicine, Lester and Sue Smith Breast Center, MS: 600, One Baylor Plaza, Room N1112, Houston, TX 77030,USA. e-mail: steffio@ breastcenter.tmc.edu

To purchase reprints of this article, contact: Reprints@BioTechniques.com

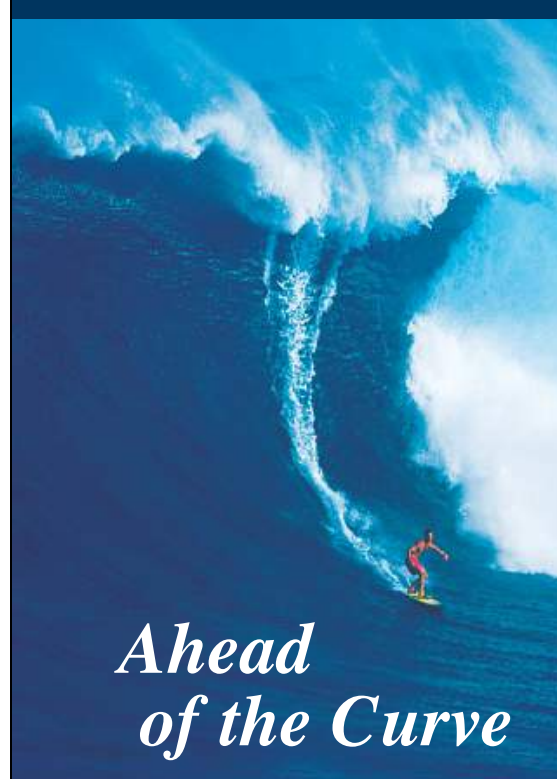

\section{Leading the Industry in Hi-Res Melting ${ }^{\mathrm{TM}}$ Curve Analysis}

Only with the LightScanner ${ }^{\circledR}$ do you get a system built by the same people who invented the technology and developed the chemistry. Idaho Technology's LightScanner System provides a complete solution for Hi-Res Melting.

Selecting Idaho Technology means gaining access to those who know the field better than anyone else.
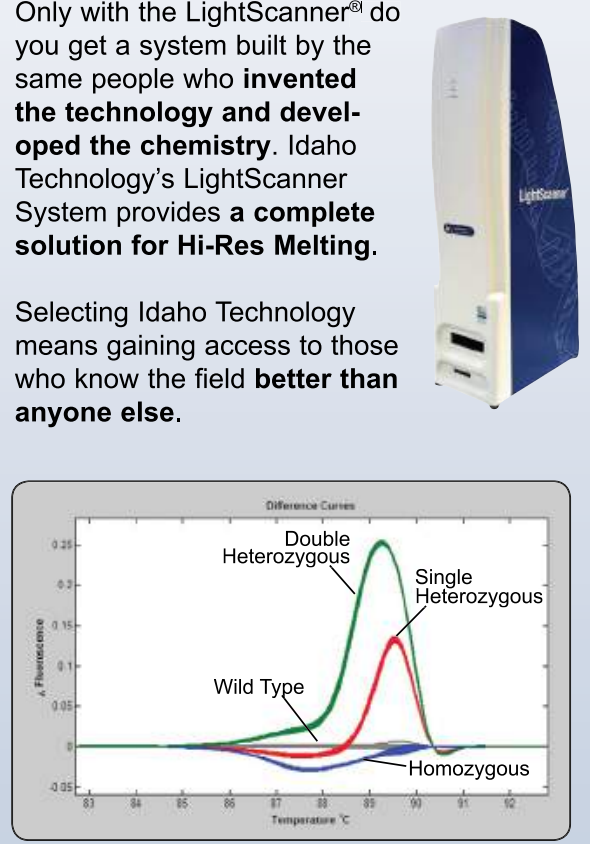

Mutation Scanning Data Analysis: Four distinct groups can be identified by the difference compared to the wild type sample.

"Designed for Hi-Res Melting" lets you know that you have chosen the RIGHT tool for the job.

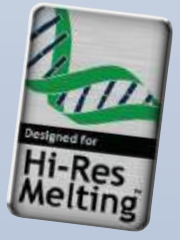

